\title{
Experiências na família de origem que repercutem no clima familiar dos descendentes
}

\section{Experiences in the family of origin that impact the family atmosphere the descendants}

\section{Experiencias en la familia de origen que impactan el ambiente familiar los descendientes}

\section{Ramona Daniela Bernardo Costa*}

Universidade do Vale do Rio dos Sinos - UNISINOS, São Leopoldo, Rio Grande do Sul, Brasil

\section{Crístofer Batista da Costa**}

Universidade do Vale do Rio dos Sinos - UNISINOS, Porto Alegre, Rio Grande do Sul, Brasil

\section{Clarisse Pereira Mosmann***}

Universidade do Vale do Rio dos Sinos - UNISINOS, São Leopoldo, Rio Grande do Sul, Brasil

\section{Denise Falcke****}

Universidade do Vale do Rio dos Sinos - UNISINOS, São Leopoldo, Rio Grande do Sul, Brasil

\begin{abstract}
RESUMO
As características do sistema familiar podem ser fatores de risco ou proteção para o desenvolvimento dos seus membros. Na família, o indivíduo constitui sua identidade e seu repertório inicial de habilidades emocionais, cognitivas e sociais que usará na vida adulta. O objetivo deste estudo foi investigar se as experiências na família de origem reverberam no clima familiar dos seus descendentes, e o poder preditivo das variáveis encontradas. Foram avaliadas 372 pessoas, sendo 186 mulheres e 186 homens. A idade dos participantes variou de 19 a 81 anos $(M=39,92$; $D P=12,62)$ e o tempo de relacionamento de 1 a 56 anos $(M=14,81$; $D P=11,69)$. Foram utilizados os questionários Family Background Questionnaire (FBQ) e Inventário do Clima Familiar (ICF). Os dados foram analisados por meio de correlação de Pearson e regressão linear método stepwise. Os resultados indicaram que todas as experiências da família de origem se correlacionaram com pelo menos uma das dimensões do clima familiar. O estilo de decisão materno e abuso de substâncias paterno vivenciados na infância e adolescência na família de origem foram as variáveis preditoras do clima familiar atual dos participantes.
\end{abstract}

Palavras-chave: relações familiares, maus tratos, criança. 


\begin{abstract}
The family system characteristics may be risk or protective factors for the development of their members. In the family, the individual shape their identity and their initial repertoire of emotional skills, cognitive and social that will use later in life. The objective of this study was to investigate the experiences in the family of origin reverberate in the family atmosphere of their descendants, the power predictive of the variables found. It were evaluated 372 people, including 186 women and 186 men. The age of participants ranged from 19 to 81 years $(M=39.92$; $S D=12.62)$ and the relationship of time $1-56$ years $(M=14.81 ; S D=11.69)$. Questionnaires Family Background Questionnaire (FBQ) and Inventory Family Climate (ICF) were used. Data were analyzed using Pearson correlation and linear regression stepwise. The results indicated that all the family of origin experiences correlated with at least one of the dimensions of the family atmosphere. The mother's decision to style and paternal abuse substances experienced in childhood and adolescence in the family of origin were the predictors of current family atmosphere the participants.
\end{abstract}

Keywords: family relations, child abuse, child.

\title{
RESUMEN
}

Las características del sistema familiar pueden ser de riesgo o factores de protección para el desarrollo de sus miembros. En la familia, el individuo constituye su identidad y su repertorio inicial de habilidades emocionales y cognitivas que va utilizar en el futuro. El objetivo de este estudio fue investigar qué experiencias en la familia de origen reverberan en el ambiente familiar de sus descendientes y el poder predictivo de las variables encontradas. Se evaluaron 372 personas, entre ellos 186 mujeres y 186 hombres. La edad de los participantes varió de 19 a 81 años $(M=39,92$; DT $=12,62)$ y el tiempo de relación de $1-56$ años $(M=14,81$, DT $=11,69)$. Se utilizaron el cuestionario Family Background Questionnaire (FBQ) y el Inventario de Clima Familiar (ICF). Los datos fueron analizados usando la correlación de Pearson y Regresión Lineal. Los resultados indicaron que todas las experiencias de la familia de origen están correlacionadas con al menos una de las dimensiones del entorno familiar. El estilo de decisión materno y el abuso de substancias paterno experimentado en la infancia y la adolescencia en la familia de origen fueron los predictores de ambiente familiar actual los participantes.

Palablas-clave: relaciones familiares, maltrato a los niños, niño.

\section{I ntrodução}

A família de origem transmite concepções de mundo que contribuem para a constituição da identidade dos seus membros, servem de referencial e asseguram a sobrevivência do grupo familiar. A transmissão de padrões e valores entre gerações dá ao indivíduo o sentimento de pertencimento e orienta os sistemas familiares subsequentes na construção de uma nova história, apesar de determinadas vivências e aprendizados se repetirem na vida adulta (Almeida, Magalhães, \& Féres-Carneiro, 2014; Bueno, Souza, Monteiro, \& Teixeira, 2013). 
A repercussão dessas vivências na fase adulta foi investigada por Curran, Ogolsky, Hazen e Bosch (2011) por meio de um estudo explicativo e longitudinal com 61 casais. Os dados foram coletados em uma cidade do sudoeste dos Estados Unidos, os participantes eram predominantemente brancos, $(83,3 \%)$, com idade média de 30 anos, tempo de casamento mínimo de um mês e máximo de 21 anos ( $m=2,58$ anos), em sua maioria com ensino superior ou pósgraduação $(66,7 \%)$ e renda familiar variando entre U\$ 45 e 60 mil dólares por ano. Foram analisadas as percepções dos participantes sobre o casamento dos seus pais no período em que se preparavam para o próprio casamento, e comparadas com medidas de conflito conjugal e estratégias de resolução sete anos depois. Os resultados confirmaram a hipótese dos autores de que a resolução dos conflitos no casamento dos participantes variou de acordo com a capacidade de insight, corroborando Paim e Falcke (2016). Os respondentes que tiveram memórias negativas da relação dos pais, porém, baixa capacidade de insight, isto é, associação dos conflitos conjugais dos pais com os do próprio casamento, tenderam a repetir os padrões negativos. De outro modo, os participantes que tiveram memórias negativas, porém, alta capacidade de insight, demonstraram esforços conscientes para evitar repetir os conflitos conjugais com seus/suas parceiros/as.

Além disso, o relacionamento do casal é o primeiro modelo de interação dos filhos, servindo de base para a construção dos padrões de relacionamento que estabelecem quando adultos. As características da família de origem preditoras dos conflitos conjugais nas relações amorosas dos seus membros adultos foram investigadas por Topham, Larson e Holman (2005) em uma amostra de 367 casais norte-americanos. Os participantes responderam 0 instrumento durante o processo de preparação para o casamento e ao completar quatro e sete anos de união. Os pesquisadores encontraram que a baixa qualidade do relacionamento e da disciplina nas relações mãefilho e do casamento dos pais foram preditoras de problemas na idade adulta. Os autores concluíram que os padrões de regulação emocional do indivíduo são estruturados durante o período da infância e da adolescência e que, portanto, a exposição a situações de violência ou a cuidados inadequados impactam o bem-estar psicológico dos filhos (Gross, 2013; Monteiro \& Maia, 2010; Thompson, 2013).

O impacto que a violência sofrida na infância provoca na vida adulta foi medida também em um estudo epidemiológico em que foram avaliados aspectos biomédicos, psicológicos e sociais (biopsicossociais) de mais de 50.000 pessoas. A pesquisa foi realizada em San Diego, nos Estados Unidos, e os participantes eram membros do Kaiser Foundation do Department of Preventive Medicine (Felitti, 2002). Foram avaliadas situações de abuso físico e sexual, 
presença de psicopatologias, como dependência de álcool e outras drogas, depressão e comportamentos suicidas em alguns cuidadores, presença de violência conjugal e de negligência física. Os resultados confirmaram evidências encontradas em outras pesquisas (Colossi, Marasca, \& Falcke, 2015; Marasca, Colossi, \& Falcke, 2013; Razera, Falcke, \& Cenci, 2014) sobre as associações entre as experiências destrutivas vivenciadas na infância, os prejuízos no desenvolvimento dos filhos e a qualidade de vida do indivíduo adulto. Entre as conclusões do estudo foi apontado que determinadas experiências adversas vivenciadas na infância não são superadas na adultez.

Entre os tipos de violência está a negligência física que ocorre quando os pais ou responsáveis demostram pouco envolvimento com a socialização da criança e não provêm adequadamente alimentação, vestuário e moradia. São cuidadores centrados excessivamente em seus próprios interesses e que não proporcionam aos filhos um ambiente familiar afetivo (Fonseca, Andrade, Santos, Cunha, \& Albuquerque, 2014). Outro estudo aponta que pais negligentes são responsáveis por significativo prejuízo no desenvolvimento dos seus filhos que poderão ter dificuldades nas suas relações afetivas e sociais na vida adulta (Rinhel-Silva, Constantino, \& Rondini, 2012).

O uso de força física pelos pais ou responsáveis caracteriza o abuso físico. De acordo com a literatura, nas famílias com história de abuso físico há menos interação entre pais e filhos que consideram os cuidadores autoritários, rígidos afetivamente e percebem as famílias como menos coesas (Colossi et al., 2015; De Antoni, 2005). Há também elevados níveis de conflito, baixos níveis de apoio, predomina o sentimento de rejeição entre os familiares e as relações parentais são marcadas principalmente por hostilidade (De Antoni, 2005).

Além disso, há situações em que o poder está centralizado permanentemente em um familiar, geralmente o abusador, que pode ser o pai ou a mãe, revelando, inclusive, relações de poder disfuncionais e desiguais entre o casal (De Antoni, 2005; Fonseca et al., 2014). Crianças que sofreram violência física no lar internalizam esses modelos e tendem a se envolver em situações de violência no seu próprio núcleo familiar (Colossi et al., 2015; Yoshihama \& Horrocks, 2010; Villas Boas, 2014).

Outra forma de violência acontece por meio do abuso sexual. $\mathrm{O}$ ato ou a relação sexual envolve interação desigual entre agressor e vítima e um modo particular de significação para cada envolvido, direta ou indiretamente. A vítima, criança/adolescente, é submissa e não compreende a natureza real da relação no contexto de tantas outras que mantêm com seus pais ou responsáveis. $O$ abusador se utiliza da confiança e da dependência da vítima e pratica atividades de natureza sexual, mediante uma conduta coercitiva, sedutora e autoritária (Penso, Costa, Almeida, \& Ribeiro, 2009). O abuso sexual 
se caracteriza por assimetria de poder, uso de força, silêncio, segredos, cumplicidade e sedução (Silva \& Falcke, 2012). Uma criança que sofreu esse tipo de abuso no contexto familiar tem maior predisposição a reproduzir essas situações quando adulto (Yoshihama \& Horrocks, 2010; Marafiga, Falcke, \& Teodoro, 2017).

O alcoolismo e o uso de outras drogas pelos pais ou responsáveis também impactam negativamente os filhos. Famílias em que um dos cuidadores é usuário de algum tipo de substância psicoativa proporcionam um ambiente de risco ao desenvolvimento dos seus membros, especialmente se crianças ou adolescentes. Pesquisas evidenciam que filhos de dependentes de álcool e outras drogas, se comparados à população em geral, têm aproximadamente de quatro a seis vezes mais probabilidade de se tornarem usuários de substância psicoativa na vida adulta (Oliveira \& Arnauts, 2011).

O aparecimento de psicopatologias na fase adulta também está relacionado à saúde mental dos pais e responsáveis durante a infância e adolescência do indivíduo (Mullick \& Goodman, 2005). A depressão materna e paterna, por exemplo, tem sido associada aos problemas emocionais dos filhos (Teodoro, Hess, Saraiva, \& Cardoso, 2014).

No estudo de Fleitlich e Goodman (2001) foram investigadas as associações entre problemas de saúde mental infantil e fatores sociais, tais como pobreza, violência familiar e doença mental dos pais. A amostra foi constituída de 898 crianças e adolescente com idades entre 7 e 14 anos, recrutados em três contextos distintos, uma favela, uma comunidade urbana e uma aldeia rural, no sudeste do Brasil. Os resultados apontaram que o desequilíbrio psicológico materno e a qualidade do relacionamento familiar foram responsáveis por $\mathbf{2 8 \%}$ dos problemas comportamentais e emocionais dos filhos, se refletindo na saúde mental destes na fase adulta.

Ademais, a exposição da criança à violência conjugal é um dos motivos que contribui para que o fenômeno se perpetue de geração em geração. Ocorre que o contexto violento se torna algo naturalizado na família, seja quando o indivíduo é vítima direta ou nos casos em que testemunha a violência interparental (Gover, Park, Tomsich, \& Jennings, 2011; Villas Boas, 2014).

No estudo de Milletich, Kelley, Doane e Pearson (2010) foram encontradas associações entre experiências de violência interparental na infância (abuso físico e emocional), vitimização e perpetração de violência física na vida adulta. Participaram do estudo 658 estudantes universitários (183 homens e 475 mulheres) que estavam em uma relação de namoro, residentes no estado de Virgínia, sudeste dos Estados Unidos. Os resultados do estudo corroboram estudos nacionais (Colossi et al., 2015; Marafiga et al., 2017; Marasca et al., 2013; Paim \& Falcke, 2016; Villas Boas 2014) de que o comportamento violento se expressa por meio da repetição de 
padrões relacionais, perpetuando-se, em alguma medida, relações violentas ao longo das gerações e demonstrando que a família de origem tem um papel determinante na transmissão transgeracional da violência conjugal.

Por outro lado, nas famílias em que as relações conjugais e parentais são funcionais e os problemas são resolvidos predominantemente via estratégias construtivas, os membros se apoiam diante das situações difíceis e prezam pelo bem comum. O ambiente familiar oferece condições adequadas para o bem-estar físico e mental de cada um dos seus integrantes e da coletividade. Quando o ajustamento psicológico e o estilo de decisão dos pais são coerentes, favorecem o desenvolvimento adequado dos filhos e a maior funcionalidade nos relacionamentos futuros, repercutindo positivamente no clima familiar dos mesmos (Monteiro \& Maia, 2010; Paim \& Falcke, 2016; Ramalho, 2015; Scorsolini-Comin, Fontaine, Barroso, \& Santos, 2016; Teodoro, 2009; Thompson, 2013).

O clima familiar é medido pela percepção que os membros de uma família têm sobre as características dos relacionamentos que estabelecem entre si e sobre o funcionamento familiar (Teodoro, Allgayer, \& Land, 2009). De acordo com Moos e Moos (1994), ele pode ser definido a partir da percepção dos integrantes de uma família sobre os relacionamentos intrafamiliares, crescimento pessoal, organização e controle do sistema.

As relações familiares podem ser compreendidas por meio da avaliação de quatro fatores que indicarão se o clima familiar está funcional ou disfuncional. As variáveis são: coesão, apoio, hierarquia e conflito (Teodoro et al., 2009). A coesão é definida como proximidade afetiva entre o casal, entre pais e filhos e entre irmãos, e está relacionada ao desenvolvimento saudável e ao bem-estar dos integrantes de uma família (De Antoni, 2005). O apoio avalia o suporte emocional e material que os membros da família dão e recebem um do outro e pode ser descrito como um conjunto de sentimentos positivos existentes entre as pessoas do núcleo familiar (Teodoro, 2009; Teodoro et al., 2009).

O fator hierarquia está associado à diferenciação clara da autoridade no ambiente familiar, sendo os adultos mais influentes nas decisões familiares que os jovens. Representa o nível de poder e de controle no contexto familiar (De Antoni, 2005). O conflito é avaliado a partir das relações entre os membros da família, se perpassadas por agressividade, crítica e divergências frequentes e intensas (Teodoro et al., 2009). Segundo Teodoro (2009) o conflito é compreendido como uma gama de sentimentos que podem ser tanto uma fonte geradora de estresse quanto de agressividade no contexto familiar.

De acordo com a literatura (Rosado, Barbosa, \& Wagner, 2016; Marasca et al., 2013) as experiências na família de origem são responsáveis pela construção de valores que orientam a vida humana 
e as relações futuras dos seus membros. Nesse sentido, o papel das memórias originadas na família de origem pode auxiliar na compreensão do clima familiar atual (Curran et al., 2011; Paim \& Falcke, 2016). Investigar se características disfuncionais de um sistema familiar são padrões de comportamento e de relacionamento transmitidos pela família de um ou ambos os cônjuges (Almeida et al., 2014; Bueno et al., 2013) pode contribuir para a avaliação dessas dinâmicas especialmente na clínica psicológica.

Além disso, é necessário considerar que há estudos em que repetições de violência foram interrompidas (Rosa, Haack, \& Falcke, 2015) ou que não encontraram associação ao investigar as relações existentes entre a percepção da conjugalidade dos pais pelos filhos casados, o bem-estar subjetivo e a satisfação com a própria conjugalidade (Scorsolini-Comin, Fontaine, Barroso, \& Santos, 2015). Portanto, identificar quais questões aprendidas na família tendem a se reproduzir na vida adulta e no novo sistema familiar e a frequência de tais repetições pode auxiliar no desenvolvimento de intervenções em nível individual, conjugal e familiar (Bueno et al., 2013; Topham et al., 2005; Marasca et al., 2013). Considerando os pressupostos apresentados, o objetivo da presente pesquisa foi investigar se as experiências na família de origem reverberam no clima familiar dos seus descendentes e o poder preditivo das variáveis encontradas.

\section{Método}

\subsection{Delineamento}

Realizou-se um estudo quantitativo e transversal, com delineamento correlacional e explicativo.

Amostra: participaram deste estudo 372 respondentes, 50\% do sexo masculino e $50 \%$ do sexo feminino, selecionados inicialmente por conveniência - pessoas da rede de contatos dos membros do grupo de pesquisa, seguindo a coleta por meio de amostragem bola de neve, em que os respondentes indicam outras pessoas. Os casais residiam na região metropolitana de Porto Alegre, eram casados oficialmente ou coabitavam pelo período mínimo de seis meses e pertenciam ao nível sócio econômico médio (renda média $=\mathrm{R} \$ 3.411,21 ; \mathrm{dp}=2.520,44)$. A idade dos participantes variou de 19 a 81 anos $(m=39,92 ; d p=12,62)$ e o tempo de relacionamento de 1 a 56 anos $(m=14,81 ; d p=11,69)$. Na tabela 1 são apresentadas frequências e percentuais que caracterizam a amostra. 


\begin{tabular}{l|l|l|l}
\multicolumn{5}{c}{ Tabela 1 - Características sociodemográficas da amostra $(N=372)$} \\
\hline \multicolumn{2}{c}{ Caractericas } & $N$ & $\%$ \\
\hline Situação conjugal & Casados oficialmente & 206 & 56,7 \\
& Morando juntos & 157 & 43,3 \\
\hline Casamento anterior & Sim & 75 & 20,4 \\
& Não & 293 & 79,6 \\
\hline Filhos & Sim & 236 & 63,8 \\
& Não & 134 & 36,2 \\
\hline Escolaridade & Sem instrução & 2 & 0,6 \\
& Fundamental & 30 & 8,6 \\
& Médio & 145 & 41,4 \\
& Superior & 173 & 49,4 \\
\hline Exerce atividade & Sim & 305 & 85 \\
remunerada & Não & 54 & 15 \\
& & & \\
\hline Religião & Católica & 251 & 69,9 \\
& Evangélica & 28 & 7,8 \\
& Protestante & 12 & 3,3 \\
& Espírita & 28 & 7,8 \\
& Outra & 18 & 5 \\
& Sem religião & 22 & 6,1 \\
\hline & Nada & 39 & 10,5 \\
& Pouco & 107 & 28,9 \\
& Médio & 129 & 34,9 \\
& Bastante & 71 & 19,2 \\
& Muito & 24 & 6,5 \\
\hline \multirow{2}{*}{ Quanto é religioso } & & &
\end{tabular}

\subsection{Instrumento}

a) Ficha de dados sócio-demográficos.

b) Family Background Questionnaire (FBQ): questionário constituído por 179 itens, distribuídos em 22 escalas. Apresenta questões sobre as memórias de experiências na família de origem até o período de 18 anos de idade. Uma escala tipo Likert de um a cinco pontos foi usada para todos os itens do questionário. Todas as pontuações foram cotadas, sendo que as mais elevadas indicaram o melhor nível de funcionamento familiar. Relativamente aos checklists, foram constituídas questões qualitativas do tipo "sim" e "não" (Monteiro \& Maia, 2010).

c) Inventário do Clima Familiar (ICF): inventário constituído por itens baseados em instrumentos internacionais, tais como o Family Climate Inventory (Kudek, Fine, \& Sinclair, 1995) e o Family Adaptability and Cohesion Evaluation Scale (Olson, Portner, \& Lavee, 1985). A elaboração das definições dos construtos orientou-se por escalas utilizadas anteriormente em outros estudos (Teodoro, 2009). O ICF tem 22 itens em escalas Likert de cinco pontos (de "não concordo" 
até "concordo plenamente"), divididos em quatro fatores: coesão, apoio, hierarquia e conflito familiar. Os resultados psicométricos apontaram estrutura fatorial compatível com o modelo de quatro fatores e Alphas de Cronbach superiores a 0,80 (Teodoro et al., 2009).

\subsection{Procedimentos éticos e de coleta de dados}

A pesquisa foi aprovada pelo Comitê de Ética da Universidade do Vale do Rio dos Sinos, sob o parecer no $11 / 129$. Os respondentes foram contatados e convidados para participar do estudo via telefone. No dia e horário agendado para aplicação do instrumento foram informados sobre as questões éticas e assinaram o Termo de Consentimento Livre e Esclarecido(TCLE). A aplicação foi realizada na residência dos participantes que responderam as questões na presença de um assistente de pesquisa.

\subsection{Análise de dados}

Os dados foram submetidos a análises estatísticas no programa Statistical Package for Social Science 20.0 (SPSS). Foi realizada análise descritiva dos resultados (médias, desvio padrão e porcentagens). Para a estatística inferencial foram analisados os critérios de supostos paramétricos (tipo de variável, tamanho da amostra, normalidade). Procedeu-se com análises de Correlação de Pearson para verificar se existia associação entre as variáveis e análise de regressão linear (Método Stepwise) para verificar o poder preditivo das experiências da família de origem no clima familiar.

\section{Resultados}

Na tabela 2 estão as correlações observadas entre as experiências na família de origem e as quatro dimensões do clima familiar. Todas as experiências da família de origem se correlacionaram com pelo menos uma das dimensões do clima familiar. 
Tabela 2. Correlações entre as dimensões do FBQ e do ICF

\begin{tabular}{l|l|l|l|l}
\hline \multicolumn{1}{c}{ Dimensões FBQ } & \multicolumn{4}{c}{ Dimensões ICF } \\
\cline { 2 - 5 } & Apoio & Hierarquia & Coesão & Conflito \\
\hline Abuso físico paterno &,- 062 &, $162^{* *}$ &,- 017 &, $163^{* *}$ \\
Abuso físico materno &,$- 123^{*}$ &, $162^{* *}$ &,- 035 &, $248^{* *}$ \\
Abuso sexual &,$- 128^{*}$ &, $243^{* *}$ &,- 105 &, $147^{*}$ \\
Negligência física &,$- 146^{*}$ &, 077 &,- 110 &, 108 \\
Estilo de decisão paterno &, 112 &,- 123 &, 111 &,$- 181^{* *}$ \\
Estilo de decisão materno &, 104 &,$- 139^{*}$ &, $156^{*}$ &,$- 222^{* *}$ \\
Abuso de substância paterna &,- 040 &, $174^{*}$ &,- 025 &, 064 \\
Abuso de substância materno &,- 017 &, 042 &,- 079 &, $157^{*}$ \\
Ajustamento psicológico paterno &, 052 &,- 088 &, 086 &,$- 165^{*}$ \\
Ajustamento psicológico materno &, $138^{*}$ &,- 130 &, $185^{* *}$ &,$- 265^{* *}$ \\
Aliança parental &, 018 &,- 084 &, 101 &,$- 202^{* *}$ \\
\hline ** $p<0,001$ & $*<0,005$
\end{tabular}

A análise de regressão múltipla avaliou as experiências na família de origem preditoras do clima familiar. As variáveis estilo de decisão materno e abuso de substâncias paterno mostraram-se preditoras do clima da família atual dos participantes (Tabela 3). Tal procedimento forneceu um coeficiente de variância explicada $\left(R^{2}\right)$ de 0,206, indicando que essas variáveis explicaram o percentual de $20,6 \%$ do clima familiar.

Tabela 3. Experiências na família de origem preditoras do clima familiar Coeficientes não Padronizados Coeficientes Padronizados

\begin{tabular}{lccccc}
\hline \multicolumn{1}{c}{ Variáveis do modelo } & $\mathrm{B}$ & $\begin{array}{c}\text { Modelo } \\
\text { Padrão }\end{array}$ & Beta & $\mathrm{T}$ & Sig \\
\hline Estilo de decisão materno & 0,492 & 0,119 & 0,398 & 4,509 & 0,000 \\
\hline Abuso de substâncias paterno & $-0,468$ & 0,145 & $-0,285$ & $-3,224$ & 0,000
\end{tabular}

$R=0,454 \quad R^{2}=0,206 \quad R^{2}$ ajustado $=0,191$

Os resultados evidenciaram o impacto das experiências da família de origem no clima familiar. O estilo de decisão materno, quanto à coerência, consistência e adequação das argumentações da mãe mostrou-se protetivo do clima familiar, enquanto o abuso de substâncias paterno interferiu negativamente no clima familiar do indivíduo adulto. 


\section{Discussão}

As experiências na família de origem avaliadas se correlacionaram com pelo menos uma das dimensões do clima familiar, sendo o conflito a dimensão que apresentou o maior número de correlações. Esse resultado pode indicar que os conflitos são reflexos dos padrões relacionais aprendidos na infância que se repetem transgeracionalmente. São formas menos eficazes de lidar com as diferenças e com os problemas familiares e constituem o repertório de vivências que o indivíduo internalizou junto a sua família de origem (Almeida et al., 2014; Bueno et al., 2013; Curran et al., 2011).

Os resultados evidenciaram que o abuso físico paterno e o abuso físico materno se correlacionaram positivamente com hierarquia e conflito e o abuso físico materno se correlacionou negativamente com apoio. Confirma-se que o uso da força física por parte dos cuidadores representa uma forma arbitrária de cuidado para com os filhos, instaurando um estilo autoritário e com altos níveis de exigência e poder (De Antoni, 2005; Fonseca et al., 2014). Crianças com histórico de violência física na família de origem tornam-se propensas a se envolver em situações violentas na vida adulta, o que impacta no clima familiar (Colossi et al., 2015; Yoshihama \& Horrocks, 2010; Villas Boas, 2014).

Além disso, o abuso físico cometido pela figura materna mostrou-se, neste estudo, associado à percepção de menos apoio pelos participantes que tiveram mães abusadoras. Considerando que 0 apoio representa a troca de sentimentos positivos e de suporte emocional entre os membros de uma família (Teodoro, 2009; Teodoro et al., 2009), este resultado pode indicar prejuízos para além do físico. Em uma perspectiva sistêmica da dinâmica que se desenvolve em torno do indivíduo, compreende-se que o abuso físico sofrido na infância é internalizado pela criança de tal forma que na vida adulta ela apresenta dificuldades para perceber suporte emocional no seu ambiente familiar e também para dar afeto ao outro (De Antoni, 2005).

O abuso sexual se correlacionou positivamente com hierarquia e conflito, e negativamente com apoio. De acordo com esses resultados, crianças e adolescentes vítimas de abuso sexual apresentam maior predisposição para constituir ambientes familiares conflituosos quando adultos (Yoshihama \& Horrocks, 2010). Podem, ainda, ter problemas com as relações de hierarquia e menor percepção de apoio familiar. Confirma-se o impacto negativo que as experiências negativas na família de origem, durante a infância, provocam na fase adulta, especialmente as vivências de abuso sexual (Marafiga et al., 2017; Penso et al., 2009; Silva \& Falcke, 2012). 
Essas associações podem indicar que a vivência de abuso sexual na infância gera sentimentos de ambivalência, já que os responsáveis por proporcionar afeto e proteção são as mesmas pessoas que abusam. Como consequência, os indivíduos adultos constituem famílias onde se repetem padrões ambivalentes. Neste estudo, os respondentes avaliaram o clima familiar como perpassado por problemas de hierarquia, caracterizando fronteiras pouco claras entre os subsistemas e confusão de papéis, e dificuldades para dar e receber suporte emocional (Marafiga et al., 2017; Penso et al., 2009; Silva \& Falcke, 2012).

Foi encontrada correlação negativa entre negligência física na família de origem e apoio. A negligência sofrida na infância pode estar associada à percepção de falta de apoio na família nuclear que o indivíduo constituiu ou, ainda, tais vivências da infância podem ter levado o indivíduo a constituir relações em que os padrões interacionais, caracterizados por falta de apoio, se repetissem (Felitti, 2002; Fonseca et al., 2014; Rinhel-Silva et al., 2012).

Os estilos de decisão paterno e materno da família de origem também repercutiram no clima familiar dos filhos na fase adulta. $O$ estilo de decisão paterno se correlacionou negativamente com 0 conflito familiar e o estilo de decisão materna se correlacionou negativamente com hierarquia e conflito e positivamente com coesão. Esses resultados indicam que o estilo de decisão dos pais pode estar associado à competência social, à assertividade e ao comportamento independente dos seus membros (Fonseca et al., 2014; Paim \& Falcke, 2016; Ramalho, 2015; Rinhel-Silva et al., 2012). Pode-se compreender, por meio dessas evidências, que os participantes se desenvolveram em famílias em que as decisões das figuras parentais eram incoerentes e inconsistentes. Tal experiência levou-os a perceber negativamente o clima familiar atual, ou seja, caracterizado por desunião e conflito, em termos da hierarquia esperada entre os integrantes da família (Monteiro \& Maia, 2010; Teodoro, 2009; Teodoro, 2009).

O abuso de substâncias psicoativas também foi indicativo de prejuízos à prole. $\mathrm{O}$ abuso paterno de algum tipo de substância teve correlação positiva com a hierarquia, enquanto o abuso materno teve correlação positiva com o conflito. Esse resultado corrobora evidências encontradas em estudos anteriores (Oliveira \& Arnauts, 2011) de que no abuso paterno prepondera um estilo parental autoritário na família de origem, repercutindo em dificuldades nas relações de hierarquia do indivíduo na fase adulta. Já o abuso de substância materno e a avaliação do clima familiar como conflituoso pelo sujeito adulto pode estar associado à deficiência, própria de uma infância com mãe abusadora de droga, de repertório emocional para resolução adequada dos conflitos. 
O ajustamento psicológico paterno apresentou correlação negativa com o conflito, enquanto o ajustamento psicológico materno se correlacionou positivamente com apoio e coesão e negativamente com conflito. As análises apontam participantes que tiveram em suas famílias de origem mãe ou pai com algum tipo de transtorno mental e, por isso, podem estar percebendo a presença de conflito na sua família nuclear e também vivenciando relações conflituosas. O ajustamento psicológico materno se apresentou como fator de proteção dos filhos que se tornaram adultos que avaliam o ambiente familiar como coeso e perpassado por apoio entre os seus membros (Fleitlich \& Goodman, 2001; Teodoro et al., 2014).

A aliança parental foi uma variável da família de origem que se correlacionou negativamente com o conflito no clima familiar do indivíduo adulto. Esse resultado aponta que a qualidade da relação entre o casal, enquanto pais, funcionou como um fator de proteção aos filhos que, na fase adulta, constituíram um núcleo familiar em que os conflitos não emergiram como um aspecto problemático na interação entre os membros (Gross, 2013; Monteiro \& Maia, 2010; Teodoro, 2009). Isso pode indicar que os respondentes percebem menor ocorrência de divergências no núcleo familiar ou conseguem resolvê-las predominantemente via estratégias construtivas (Gover et al., 2011; Villas Boas, 2014; Ramalho, 2015; Scorsolini-Comin et al., 2016).

Foram avaliadas também as experiências na família de origem que predisseram mais fortemente o clima familiar atual dos participantes. As análises indicaram que o estilo de decisão materno e o abuso de substâncias paterno foram preditores do clima familiar, explicando $20,6 \%$ da variância.

O estilo de decisão materno teve predição positiva no clima familiar. Este resultado pode apontar que a coerência e a consistência nas decisões da figura materna reverberam de forma positiva no desenvolvimento dos filhos, mostrando-se como um fator protetivo na vida adulta (Fleitlich \& Goodman, 2001; Rosa et al., 2015; Teodoro et al., 2014). Também, que o papel parental da mulher é significativo na educação da prole (Topham et al., 2005) e pode estar associado ao fato de a mãe permanecer mais tempo e se envolver mais efetivamente nos cuidados com os filhos do que o pai. Observase que, embora essa dinâmica venha sofrendo alterações nos dias atuais, o envolvimento da mãe nos cuidados com os filhos ainda prepondera.

O abuso de substâncias pela figura paterna, preditor negativo do clima familiar, indica que o abuso de drogas pelo progenitor ou responsável do sexo masculino caracteriza um contexto extremamente deletério a saúde dos seus membros, especialmente dos filhos. Pode-se compreender, por meio dessa evidência, que o abuso de droga pelo progenitor é traumático à criança ou 
adolescente, prejudicando suas relações na fase adulta e sua percepção do clima na família constituída (Oliveira \& Arnauts, 2011; Yoshihama \& Horrocks, 2010).

\section{Considerações finais}

De acordo com os resultados encontrados neste estudo, corroborando com a literatura científica da área, observa-se que efetivamente as vivências de crianças e adolescentes na família de origem reverberam na vida adulta. Compreende-se que essas experiências podem constituir fatores de risco ou de proteção à prole, condição que dependerá da capacidade dos pais de assegurar bem-estar aos filhos e manter relações de respeito entre si.

Além disso, as evidências que emergiram desta investigação sugerem que a história familiar de um adulto que foi vítima ou testemunha de vivências infantis perpassadas por violência intrafamiliar, envolvimento dos pais com substâncias psicoativas ou problemas de psicopatologia gera consequências na constituição da sua própria família.

A realização deste estudo retifica a relevância de se permanecer investigando a temática em questão. Identificar as repercussões em decorrência dos diferentes tipos de abuso sofridos na família de origem, os quais provocam psicopatias na vida adulta, pode contribuir para a compreensão do clima familiar na clínica psicológica individual, de casal e família. Ademais, a clareza acerca da reverberação das experiências infantis em longo prazo pode contribuir para 0 desenvolvimento de intervenções com o objetivo de interromper a repetição de padrões interacionais violentos ou perpassados por outros tipos de abuso.

Enquanto limitações desta pesquisa, citam-se o corte transversal e a utilização de apenas dois instrumentos, um para avaliar experiências da família de origem e outro para avaliar o clima familiar. Considerando a complexidade do fenômeno investigado, pesquisas longitudinais e utilizando mais instrumentos para avaliar o fenômeno de diferentes formas poderão fornecer dados mais robustos acerca das variáveis familiares que interferem no contexto familiar do indivíduo adulto. Além disso, é importante ressaltar que outras variáveis, como características individuais e contextuais e a atualização das experiências na conjugalidade construída na vida adulta podem interferir no clima familiar e, portanto, necessitam ser investigadas. Finalmente, estudos por meio de método qualitativo poderiam contribuir para a compreensão de especificidades da vivência de indivíduos vítimas de algum tipo de violência durante a infância e adolescência, avaliando, por exemplo, a percepção dessas 
pessoas e a forma como gerenciaram tais experiências em suas vidas na fase adulta.

\section{Referências}

Almeida, M. E., Magalhães, A. S., \& Féres-Carneiro, T. (2014). Transmissão geracional da profissão na família: Repetição e diferenciação. Psico, 45(4), 454-462.

Bueno, R. K., Souza, S. A., Monteiro, M. A., \& Teixeira, R. H. M. (2013). Processo de diferenciação dos casais de suas famílias de origem. Psico, 44(1), 16-25.

Colossi, P. M., Marasca, A. R., \& Falcke, D. (2015). De geração em geração: A violência conjugal e as experiências na família de origem. Psico, 46(4), 493-502.

Curran, M., Ogolsky, B., Hazen, N., \& Bosch, L. (2011). Understanding marital conflict 7 years later from prenatal representations of marriage. Family Process, 50(2), 221-234. doi: $10.1111 / \mathrm{j} .1545-5300.2011 .01356 . x$

De Antoni, C. (2005). Coesão e hierarquia em famílias com história de abuso físico (Tese de Doutorado). Retirado de http: // www. lume.ufrgs. br/ handle/10183/6137

Felitti, V. J. (2002). The relationship of adverse childhood experiences to adult health: Turning gold into lead. The Permanente Journal, 6(1), 44-47.

Fehringer, J. A., \& Hindin, M. J. (2009). Like parent, like child: Intergenerational transmission of partner violence in Cebu, the Philippines. Journal of Adolescent Health, 44(4), 363-371. doi: 10.1016/j.jadohealth.2008.08.012

Fleitlich, B., \& Goodman, R. (2001). Social factors associated with child mental health problems in Brazil: Cross sectional survey. British Medical Journal, 323(7313), 599-600. doi: $10.1136 / \mathrm{bmj} .323 .7313 .599$

Fonseca, P. N., Andrade, P. O., Santos, J. L. F., Cunha, J. E. M., \& Albuquerque, J. H. A. (2014). Hábitos de estudo e estilos parentais: Estudo correlacional. Psicologia Escolar e Educacional, 18(2), 337-345. doi: 10.1590/21753539/2014/0182755

Gover, A. R., Park, M., Tomsich, E. A., \& Jennings, W. G. (2011). Dating violence perpetration and victimization among South Korean college students: A focus on gender and childhood maltreatment. Journal of Interpersonal Violence, 26(6), 123263. doi: $10.1177 / 0886260510368161$

Gross, J. J. (2013). Emotion regulation: conceptual and empirical foundations. In J. J. Gross (Ed.). Handbook of emotion regulation (2. ed., pp. 3-20). New York, NY: Guilford Press. 
Kurdek, L. A., Fine, M. A., \& Sinclair, R. J. (1995). School Adjustment in Sixth Graders: Parenting Transitions, Family Climate, and Peer Norm Effects. Child Development, 66, 430-445. doi: $10.2307 / 1131588$

Marafiga, C. V., Falcke, D., \& Teodoro, M. L. M. (2017). Pedofilia: História de vida e o retorno para a família por meio de alta progressiva. Revista da SPAGESP, 18(1), 48-6.

Marasca, A. R., Colossi, P. M., \& Falcke, D. (2013). Violência conjugal e família de origem: Uma revisão sistemática da literatura de 2006 a 2011. Temas em Psicologia, 21(1), 221-243.

Milletich, R. J., Kelley, M. L., Doane, A. N., \& Pearson, M. R. (2010). Exposure to interparental violence and childhood physical and emotional abuse as related to physical aggression in undergraduate dating relationships. Journal of Family Violence, 25(7), 627-637. doi: 10.1007/s10896-010-9319-3

Monteiro, I. S., \& Maia, A. C. (2010). Avaliação psicométrica de três questionários sobre o historial familiar. Revista de Psiquiatria Clínica, 37(3), 97-104. doi: 10.1590/S010160832010000300001

Moos, R. H., \& Moos, B. S. (1994). Family Environment Scale Manual. (3. ed.). Palo Alto, CA: Consulting Psychologists Press.

Mullick, M. S. I., \& Goodman, R. (2005). The prevalence of psychiatric disorders among 5-10 year olds in rural, urban and slum areas in Bangladesh. Social Psychiatry and Psychiatric Epidemiology, 40(8), 663-671. doi: 10.1007/s00127-005-09395

Oliveira, M. L. F., \& Arnauts, I. (2011). Intoxicação alcoólica em crianças e adolescentes: Dados de um centro de assistência toxicológica. Escola Anna Nery, 15(1), 83-89. doi: 10.1590/S1414-81452011000100012

Olson, D. H., Portner, J., \& Lavee, Y. (1985). "Faces III": Family Adaptability \& Cohesion Evaluation Scales, Family Social Science. St. Paul: University of Minnesota.

Paim, K., \& Falcke, D., (2016). Perfil discriminante de sujeitos com histórico de violência conjugal: $O$ papel dos esquemas iniciais desadaptativos. Revista Brasileira de Terapia Comportamental e Cognitiva, 18(2), 112-129.

Penso, M. A., Costa, L. F., Almeida, T. M. C., \& Ribeiro, M. A. (2009). Abuso sexual intrafamiliar na perspectiva das relações conjugais e familiares. Aletheia, 30, 142-157.

Ramalho. A. C. F. N. P. (2015). Relação do envolvimento paterno com variáveis do pai, da criança, da família de origem e da relação conjugal (Dissertação de Mestrado). Universidade de Lisboa, Lisboa, Portugal.

Razera, J., Falcke, D., \& Cenci, C. M. B. (2014). Violência doméstica e transgeracionalidade: Um estudo de caso. Revista de Psicologia 
da IMED, 6(1), 47-51. doi: 10.18256/2175-5027/psicoimed.v6n1p47-51

Rinhel-Silva, C. M., Constantino, E. P., \& Rondini, C. A. (2012). Família, adolescência e estilos parentais. Estudos de Psicologia, 29(2), 221-230. doi: 10.1590/S0103-166X2012000200008

Rosado, J. S., Barbosa, P. V., \& Wagner, A. (2016). Ajustamento conjugal: A função das características individuais, do casal e do contexto. Psicologia em Pesquisa, 10(1), 26-33.

Rosa, L. W., Haack, K. R., \& Falcke, D. (2015). Rompendo o ciclo de violência na família: Concepções de mães que não reproduzem - abuso sofrido na infância com seus filhos. Revista de Psicologia da IMED, 7(2), 26-36. doi: 10.18256/21755027/psico-imed.v7n2p26-36

Silva, N. B., \& Falcke, D. (2012). Família incestuosa: Do silêncio à possibilidade de revelação. Pensando Famílias, 16(1), 177-197.

Scorsolini-Comin, F., Fontaine, A. M. G. V., Barroso, S. M., \& Santos, M. A. (2015). Relações entre conjugalidade dos pais, conjugalidade dos filhos e bem-estar subjetivo. Psico-USF, 20(3), 481-492.

Scorsolini-Comin, F., Fontaine, A. M. G. V., Barroso, S. M., \& Santos, M. A. (2016). Fatores associados ao bem-estar subjetivo em pessoas casadas e solteiras. Estudos de Psicologia (Campinas), 33(2), 313-324.

Teodoro, M. L. M. (2009). Família, bem-estar e qualidade de vida de crianças e adolescentes. In V. G. Haase, F. O. Ferreira, \& F. J. Penna (Eds.), Aspectos biopsicossociais da saúde na infância e adolescência (pp. 111-112). Belo Horizonte: Coopmed.

Teodoro, M. L. M., Allgayer, M., \& Land, B. (2009). Desenvolvimento e validade fatorial do Inventário do Clima Familiar (ICF) para adolescentes. Psicologia: Teoria e Prática, 11(3), 27-39. Retirado de http://editorarevistas. mackenzie. br/index.php/ptp/article/view/ 2223/1988

Teodoro, M. L. M., Hess, A. R. B., Saraiva, L. A., \& Cardoso, B. M. (2014). Problemas emocionais e de comportamento e clima familiar em adolescentes e seus pais. Psico, 45(2), 168-175. doi: 10.15448/1980-8623.2014.2.13172

Thompson, R. A. (2013). Socialization of emotion and emotion regulation in the family. In: J. J. Gross (Ed.). Handbook of emotion regulation (2. ed., pp. 173-186). New York, NY: Guilford Press.

Topham, G. L., Larson, J. H., \& Holman, T. B. (2005). Family-oforigin predictors of hostile conflict in early marriage. Contemporary Family Therapy, 27(1), 101-121. doi: 10.1007/s10591-004-1973-2 
Villas Boas, A. C. V. B. (2014). Violência física contra a criança: Fatores de risco e proteção e padrões de interação na família (Tese de Doutorado). Universidade de Brasília, Brasília, Brasil.

Yoshihama, M., \& Horrocks, J. (2010). Risk of intimate partner violence: Role of childhood sexual abuse and sexual initiation in women in Japan. Children and Youth Services Review, 32(1), 28-37. doi: 10.1016/j.childyouth.2009.06.013

\section{Endereço para correspondência}

Ramona Daniela Bernardo Costa

Universidade do Vale do Rio dos Sinos

Avenida Unisinos, 950, Cristo Rei, CEP 93022-750, São Leopoldo - RS, Brasil

Endereço eletrônico: dbc_ramona@gmail.com

Crístofer Batista da Costa

Universidade do Vale do Rio dos Sinos

Avenida Unisinos, 950, Cristo Rei, CEP 93022-750, São Leopoldo - RS, Brasil

Endereço eletrônico: cristoferbatistadacosta@gmail.com

\section{Clarisse Pereira Mosmann}

Universidade do Vale do Rio dos Sinos

Avenida Unisinos, 950, Cristo Rei, CEP 93022-750, São Leopoldo - RS, Brasil

Endereço eletrônico: clarissemosmann@gmail.com

\section{Denise Falcke}

Universidade do Vale do Rio dos Sinos

Avenida Unisinos, 950, Cristo Rei, CEP 93022-750, São Leopoldo - RS, Brasil

Endereço eletrônico: dfalcke@unisinos.br

Recebido em: 18/01/2016

Reformulado em: 29/09/2017

Aceito em: 29/09/2017

\section{Notas}

* Psicóloga. Especialista em Terapia Sistêmica.

** Psicólogo. Mestre e Doutorando em Psicologia Clínica.

*** Psicóloga. Doutora em Psicologia (PUC/RS).

**** Psicóloga. Doutora em Psicologia Clínica (PUC/RS).

Este artigo de revista Estudos e Pesquisas em Psicologia é licenciado sob uma Licença Creative Commons Atribuição-Não Comercial 3.0 Não Adaptada. 